\title{
Endoscopic-assisted interhemispheric parieto-occipital transtentorial approach for microsurgical resection of a pineal region tumor: operative video and technical nuances
}

\begin{abstract}
James K. Liu, MD
Department of Neurological Surgery, Center for Skull Base and Pituitary Surgery, Neurological Institute of New Jersey, Rutgers New Jersey Medical School, Newark, New Jersey

The angle of the straight sinus and tentorium cerebelli can often influence the choice of surgical approach to the pineal region. The supracerebellar infratentorial approach can be technically challenging and a relative contraindication in cases where the angle of the straight sinus and tentorium is very steep. Similarly, an occipital transtentorial approach, which uses a low occipital craniotomy at the junction of the superior sagittal sinus and transverse sinus, may not provide the best trajectory to the pineal region in patients with a steep tentorium. In addition, this approach often necessitates retraction on the occipital lobe to access the tentorial incisura and pineal region, which can increase the risk of visual compromise. In this operative video, the author demonstrates an alternative route using an endoscopic-assisted interhemispheric parieto-occipital transtentorial approach to a pineal region tumor in a patient with a steep straight sinus and tentorium. The approach provided a shorter route and more direct trajectory to the tumor at the tentorial incisura, and avoided direct fixed retraction on the occipital lobe when performed using the lateral position, thereby minimizing visual complications. This video atlas demonstrates the operative technique and surgical nuances, including the application of endoscopic-assisted microsurgical resection and operative pearls for preservation of the deep cerebral veins. In summary, the parieto-occipital transtentorial approach with endoscopic assistance is an important approach in the armamentarium for surgical management of pineal region tumors.
\end{abstract}

The video can be found here: https://youtu.be/Ph4veG14aTk.

KEYWORDS parieto-occipital transtentorial approach; endoscopic-assisted microsurgery; interhemispheric; pineal region tumor; video

SUBMITTED August 31, 2015. ACCEPTED September 30, 2015.

INCLUDE WHEN CITING Published online January 1, 2016; DOI: http://thejns.org/doi/abs/10.3171/2016.1.FocusVid.15450.

CORRESPONDENCE James K. Liu, MD, Department of Neurological Surgery, Rutgers University, New Jersey Medical School, 90 Bergen Street, Suite 8100, Newark, New Jersey 07103. email: james.liu.md@rutgers.edu. 